\title{
Composition and richness of the serum microbiome differ by age and link to systemic inflammation
}

\author{
Thomas W. Buford (i) - Christy S. Carter • William J. VanDerPol • Dongquan Chen • \\ Elliot J. Lefkowitz • Peter Eipers • Casey D. Morrow • Marcas M. Bamman
}

Received: 8 May 2018 / Accepted: 25 May 2018 /Published online: 5 June 2018

(C) The Author(s) 2018

\begin{abstract}
Advanced age has been associated with alterations to the microbiome within the intestinal tract as well as intestinal permeability (i.e., "leaky gut"). Prior studies suggest that intestinal permeability may contribute to increases in systemic inflammation - an aging hallmark - possibly via microorganisms entering the circulation. Yet, no studies exist describing the state of the circulating microbiome among older persons. To compare microbiota profiles in serum between healthy young (20-35 years, $n=24)$ and older adults $(60-75$ years, $n=$ 24 ) as well as associations between differential microbial populations and prominent indices of age-related inflammation. Unweighted Unifrac analysis, a measure of $\beta$ diversity, revealed that microbial communities clustered differently between young and older adults. Several measures of $\alpha$-diversity, including chao1 $(p=0.001)$, observed species $(p=0.001)$, and phylogenetic diversity
\end{abstract}

T. W. Buford $(\square) \cdot$ C. S. Carter

Department of Medicine, University of Alabama at Birmingham, Birmingham, AL 35294, USA

e-mail: twbuford@uabmc.edu

W. J. VanDerPol · D. Chen · E. J. Lefkowitz

Biomedical Informatics, Center for Clinical and Translational Sciences, University of Alabama at Birmingham, Birmingham, AL, USA

\section{E. J. Lefkowitz}

Department of Microbiology, University of Alabama at

Birmingham, Birmingham, AL, USA

P. Eipers · C. D. Morrow • M. M. Bamman

Department of Cell, Developmental, and Integrative Biology,

University of Alabama at Birmingham, Birmingham, AL, USA $(p=0.002)$ differed between young and older adults. After correction for false discovery rate (FDR), age groups differed (all $p$ values $\leq 0.016$ ) in the relative abundance of the phyla Bacteroidetes, SR1, Spirochaetes, Bacteria_Other, TM7, and Tenericutes. Significant positive correlations ( $p$ values $\leq 0.017$ after FDR correction) were observed between IGF1 and Bacteroidetes $(\rho=0.380)$, Spirochaetes $(\rho=0.528)$, SR1 $(\rho=0.410)$, and TM7 $(\rho=0.399)$. Significant inverse correlations were observed for IL6 with Bacteroidetes $(\rho=-0.398)$ and TM7 $(\rho=-0.423)$, as well as for TNF $\alpha$ with Bacteroidetes $(\rho=-0.344)$. Similar findings were observed at the class taxon. These data are the first to demonstrate that the richness and composition of the serum microbiome differ between young and older adults and that these factors are linked to indices of age-related inflammation.

Keywords Aging $\cdot$ Leaky gut $\cdot$ Microbiome .

Microbiota $\cdot$ Inflammation

\section{Introduction}

Chronic low-grade inflammation is one of the most consistent biologic features of advanced age, evidenced by over 10,000 publications in this area (Buford 2017). Yet, despite the common recognition of the inflammatory phenomenon, the etiology of agerelated inflammation remains poorly understood. Recently, a novel hypothesis has emerged from our group and others suggesting that increases in gut 
permeability (i.e., "leaky gut") and subsequent release of intestinal contents into the circulation may be a primary contributor to increases in age-related inflammation (Buford 2017; Nicoletti 2015).

Aging is associated with several relevant changes to overall gut health including increases in intestinal permeability (Man et al. 2015; Nicoletti 2015) as well as changes to the stability of the gut microbiome (Biagi et al. 2010; Jeffery et al. 2016) — the aggregate genetic material of microorganisms residing within the intestinal tract which contribute to regulating host health (Human Microbiome Project Consortium 2012). These changes are relevant in the present context as recent evidence indicates that changes in microbial composition and density can alter immunity and inflammation distal to the intestine (Belkaid and Naik 2013). Indeed, early studies in humans reported cross-sectional associations between gut microbiome profiles and circulating inflammatory cytokines of older adults (Claesson et al. 2012; Rampelli et al. 2013). However, the mechanisms through which gut dysbiosis could contribute to chronic, low-grade inflammation were unclear.

Basic and pre-clinical studies have also suggested that intestinal permeability, coupled with altered microbiota profiles (Clark et al. 2015; Rera et al. 2012), may drive age-related increases in systemic inflammation. Very recently, Thevaranjan et al. (2017) published a seminal study in a mouse model definitively demonstrating that age-related gut microbial dysbiosis drives intestinal permeability, microbial translocation to the circulation, and ultimately systemic inflammation. Yet, despite these important pre-clinical studies, data are lacking to link intestinal permeability to inflammation in humans.

We recently published the first human evidence demonstrating that circulating concentrations of zonulin, a physiologic regulator of intestinal permeability, were higher-indicating greater permeability-among healthy older adults than younger peers (Qi et al. 2017). Furthermore, zonulin concentrations were positively associated with circulating concentrations of inflammatory cytokines tumor necrosis factor alpha $(\mathrm{TNF} \alpha)$ and interleukin 6 (IL6) (Qi et al. 2017), two of the primary inflammatory cytokines consistently associated with the aging process. The objective of this study was to expand upon these findings by providing the first data comparing microbial DNA profiles within the circulation of healthy and older adults. We hypothesized that the microbiome found within serum would display age-related differences in measures of both alpha- and beta-diversity-key measures to detect differences in microbiomes between differing populations (Kumar et al. 2014). Moreover, we also aimed to identify specific microbial DNA abundances significantly associated with circulating concentrations of IL6 and TNF $\alpha$ as well as insulin-like growth factor 1 (IGF1) - a hormone known to be intricately related to inflammatory cytokine production (Maggio et al. 2013; Rajpathak et al. 2008) and recently reported to be stimulated by microbiota (Yan et al. 2016).

\section{Results}

Participant characteristics, diet, and inflammatory parameters Data from a total of 48 participants was included in the study. Participant descriptive statistics are shown in Table 1 . The young $(n=24)$ and older adult $(n=24)$ groups were balanced for sex. Participants in each group were of similar height and body mass,

Table 1 Participant demographic characteristics and inflammatory parameters

\begin{tabular}{|c|c|c|}
\hline & Young adults & Older adults \\
\hline Age, years & $27.8 \pm 4.0$ & $63.9 \pm 3.2 * *$ \\
\hline Female, $n$ & $14(58.3 \%)$ & $14(58.3 \%)$ \\
\hline Height, $\mathrm{cm}$ & $170.2 \pm 11.0$ & $169.1 \pm 10.9$ \\
\hline Body mass, $\mathrm{kg}$ & $72.7 \pm 12.9$ & $74.9 \pm 15.0$ \\
\hline Body mass index, $\mathrm{kg} / \mathrm{m}^{2}$ & $25.0 \pm 3.0$ & $25.9 \pm 3.2$ \\
\hline Body fat, $\%$ & $30.1 \pm 10.4$ & $35.9 \pm 6.4^{*}$ \\
\hline $\mathrm{VO}_{2} \max , \mathrm{mL} / \mathrm{O}_{2} / \min$ & $37.5 \pm 8.4$ & $27.0 \pm 4.8^{* *}$ \\
\hline \multicolumn{3}{|l|}{ Dietary intake } \\
\hline Total intake, kcal/day & $2003 \pm 779$ & $1767 \pm 625$ \\
\hline Carbohydrate, g/day & $253.9 \pm 103.6$ & $210.2 \pm 75.5^{*}$ \\
\hline Fiber, g/day & $20.4 \pm 8.3$ & $16.2 \pm 8.7$ \\
\hline Fat, g/day & $76.5 \pm 41.2$ & $67.8 \pm 36.4$ \\
\hline Protein, g/day & $75.4 \pm 33.9$ & $73.1 \pm 30.1 *$ \\
\hline \multicolumn{3}{|l|}{ Serum inflammatory parameters } \\
\hline Interleukin $6, \mathrm{pg} / \mathrm{mL}$ & $0.38 \pm 0.19$ & $0.52 \pm 0.20 *$ \\
\hline $\begin{array}{l}\text { Tumor necrosis factor } \alpha \text {, } \\
\mathrm{pg} / \mathrm{mL}\end{array}$ & $2.02 \pm 0.60$ & $2.20 \pm 0.44$ \\
\hline $\begin{array}{l}\text { Insulin-like growth factor } 1 \text {, } \\
\mu \mathrm{g} / \mathrm{L}\end{array}$ & $365.4 \pm 129.2$ & $188.4 \pm 82.3^{* *}$ \\
\hline
\end{tabular}


resulting in a similar mean body mass index between groups. Body fat percentage and fitness were significantly different $(p<0.05)$ between groups. Regarding dietary intake, a trend toward significance $(p=0.061)$ was observed for greater daily caloric intake among younger adults compared to older adults. Young adults consumed significantly more carbohydrate (mean difference: $43.6 \mathrm{kcal} /$ day, $p=0.008$ ), including significantly more fiber (mean difference: $4.2 \mathrm{~g} / \mathrm{day}, p=0.005$ ) than older adults. No differences were observed in daily intake of fat or protein nor in specific sub-types of fats including cholesterol, saturated fat, or mono/ polyunsaturated fats (data not shown). Serum concentrations of IL6, TNF $\alpha$, and IGF1 are shown by group in Table 1. Significant group differences $(p<0.05)$ were observed for IL6 and IGF1, but not TNF $\alpha$.

Microbial analyses-overall microbiome composition, $\beta$-diversity, and $\alpha$-diversity Figure 1 depicts the overall composition of the serum microbiomes among both young and older adults at both the phylum (A) and class (B) levels of taxonomy. Principal coordinate analysis (PCoA) revealed that age groups differed in the overall serum microbiota community structure as determined by Unweighted UniFrac (C). Key measures of $\alpha$-diversity, including richness (chaol and observed species) and phylogenetic diversity, were significantly different between young and older adults (Fig. 2). Overall sample diversity, measured according to the Shannon and
Simpson metrics, did not significantly differ between age groups.

Age-related differences in microbial abundances The relative abundance of several bacterial phyla was significantly different between age groups (Fig. 3). After correction for false discovery rate (FDR), significant group differences were observed for the phyla Bacteroidetes, SR1, Spirochaetes, Bacteria_Other, TM7, and Tenericutes. At the class level, significant group differences were observed for Bacteroidia, Mollicutes, Bacteria_Other_Other, Cytophagia, Firmicutes_Other, and Leptospirae (Table 2). Additionally, several other families with $\mathrm{p}$ values $<0.05$ but not significant after FDR correction were identified, including Erysipelotrichi, Fusobacteria, SRI_unknown, and Acidimicrobiia.

Associations of identified microbial communities with inflammatory parameters Several phyla were significantly associated with serum inflammatory parameters (Fig. 4), in particular Bacteriodetes which was significantly correlated with all three measures. The phylum TM7 was significantly correlated with both IGF1 and IL6. Additionally, several other phyla displayed $p$ values $<0.05$ but were not significant after FDR correction. These included the following: Bacteria_Other with IGF1 $(\rho=0.329, p=0.025)$, Tenericutes with IGF1 $(\rho=0.303, p=0.041)$, and Spirochaetes with TNF $\alpha$ $(\rho=-0.285, p=0.050)$. At the class level, three
Fig. 1 Taxonomic distribution of serum microbiome of healthy young and older adults by phylum (a) and class (b). c Comparison of serum microbiome $\beta$-diversity (Unweighted UniFrac) between healthy young (blue) and older (red) adults
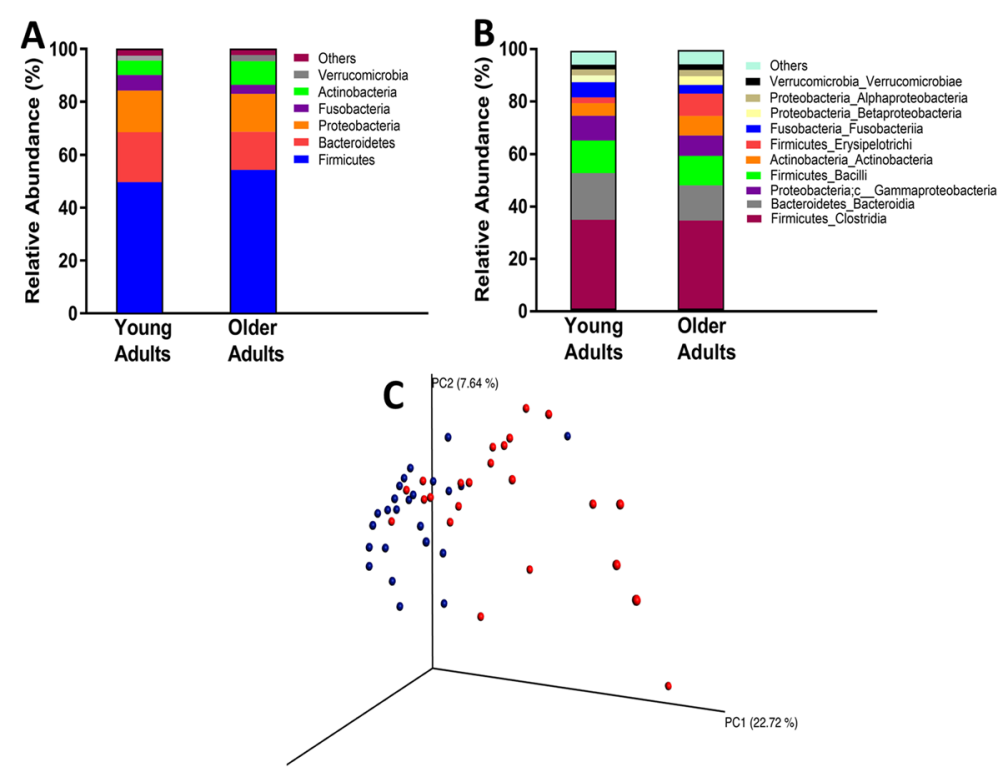

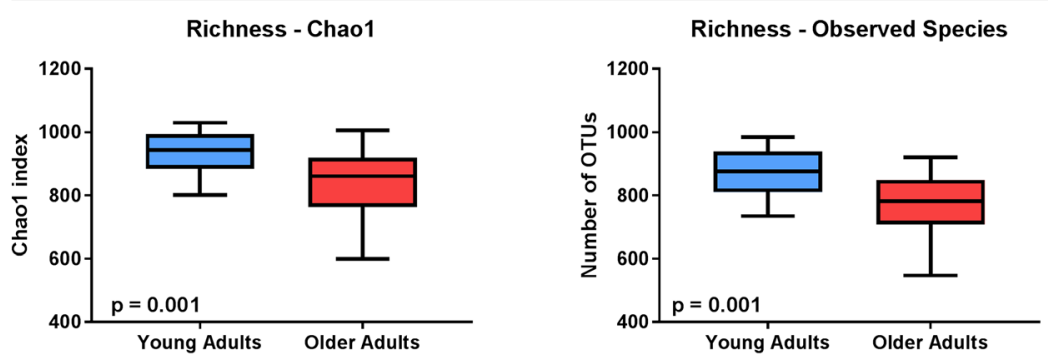

Phylogenetic Diversity

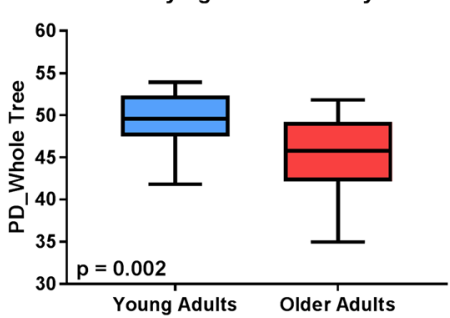

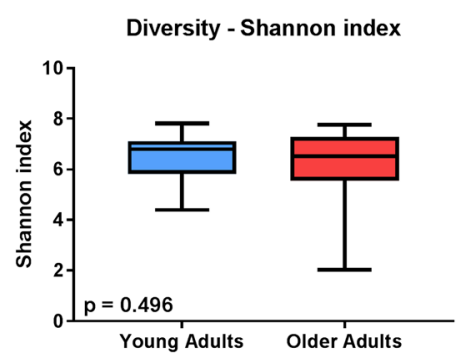

Fig. 2 Comparison of $\alpha$-diversity of the serum microbiome between healthy young (blue) and older adults (red). Five indices were used to represent the richness (chao1, observed species),

significant correlations were observed including Bacterioidia with both IGF1 and IL6 as well as Cytophagia with IGF1 (Fig. 5). Correlations with other families with $p$ values $<0.05$ but not significant after FDR correction included Bacteria_Other_Other with IGF1 $(\rho=0.318, p=0.033)$, Leptospirae with IGF1 $(\rho=0.321, p=0.031)$, and Bacterioidia with TNF $\alpha$ $(\rho=-0.324, p=0.026)$.

\section{Discussion}

This is the first study to evaluate the age-related differences in microbial DNA profiles present in serum of healthy humans as well as associations of DNA abundances of specific microbial communities with indices of systemic inflammation. These findings are the first to indicate that the community structure of the microbiome in human serum differs between healthy young and older adults. Compared to younger adults, serum of older adults contained DNA from fewer species representing a lower level of phylogenetic diversity than that of young adults. Numerous bacterial phyla- and class-level differences were observed between age groups. Notably, the relative abundance of DNA from the Bacteroidetes phylum - one of the most abundant

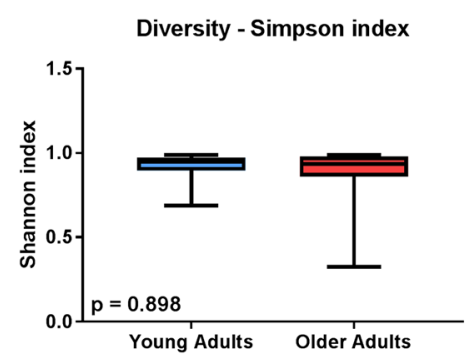

phylogenetic diversity, and sample diversity (shannon and simpson indices). Box whiskers indicate the range of observed values

bacteria in both the gut and circulation - was significantly lower among older adults. Several of these differentially expressed bacterial DNA were also significantly correlated with indices of inflammation. DNA from Bacteriodetes in particular displayed strong relationships with inflammatory parameters as it was positively associated with IGF1 and negatively associated with both IL6 and TNF $\alpha$.

Under healthy conditions, the compartmentalization of bacteria and other microbes to the gastrointestinal tract is maintained by a tight barrier at the intestinalvascular interface (Spadoni et al. 2015). Yet, under certain clinical conditions, the integrity of this barrier can decrease and result in microbial translocation to the systemic circulation. For instance, microbial translocation due to a loss of immune control has been reported in HIV+ patients (Brenchley et al. 2006) as well as in cirrhotic patients with ascites (Santiago et al. 2016). In the case of the HIV+ population, microbial translocation was associated with low-grade systemic inflammation similar to findings observed in the in recent animal study of aging (Thevaranjan et al. 2017).

In the present study, the analysis performed from whole serum cannot differentiate between microbial DNA fragments and intact microbes. Even under healthy conditions, human blood contains bacterial DNA capable of triggering host innate immune 


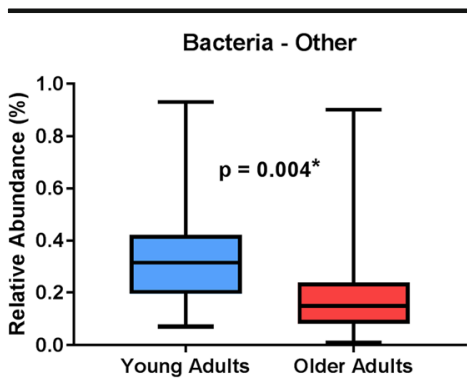

Gemmatimonadetes

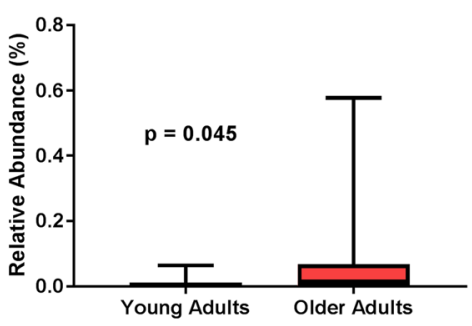

Spirochaetes

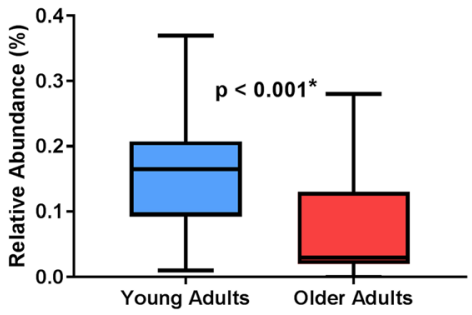

Fig. 3 Microbial DNA populations differentially expressed between young (blue) and older (red) adults at the phylum level. Asterisk indicates statistical significance after correcting for
Bacteroidetes

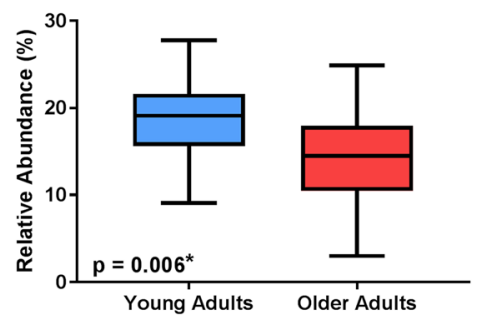

Planctomycetes

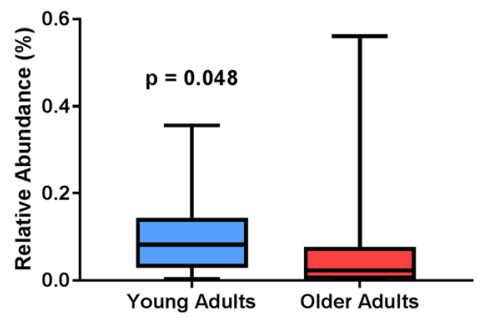

TM7

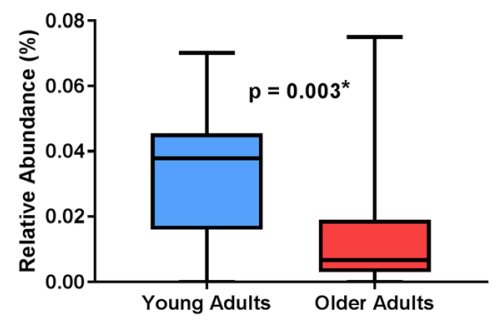

Fusobacteria

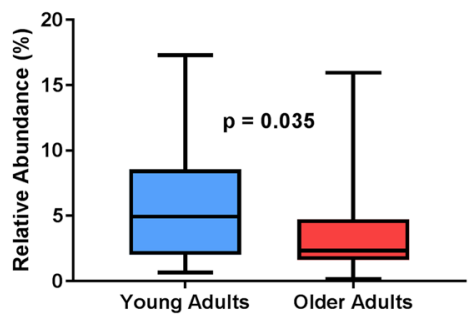

SR1

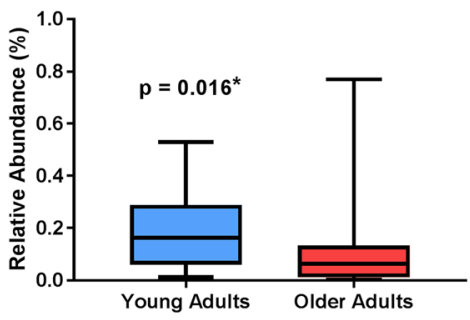

Tenericutes

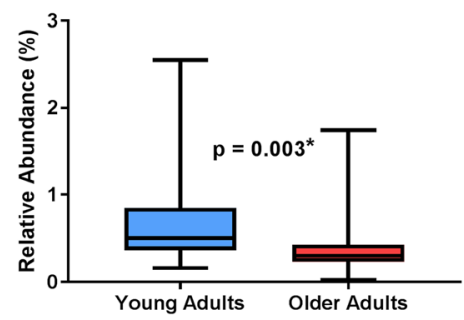

multiple comparisons via false discovery rate. Box whiskers represent the range of observed values

responses (Hacker et al. 2002; Muruve et al. 2008; Nikkari et al. 2001). What is notable here, however, is the differences in the relative abundances between young and older adults. Several studies have reported alterations in circulating bacterial DNA abundances and corresponding immune/inflammatory profiles in patient populations including those with cirrhosis, kidney disease, and cardiovascular disease (Dinakaran et al. 2014; Frances et al. 2004; Kwan et al. 2013). In fact, differences in relative bacterial DNA abundances between patients and controls were proposed as an indicator of cirrhosis progression (Santiago et al. 2016). Though we cannot confirm the cause of these differentially expressed DNA, our prior findings related to zonulin concentrations in older adults as well as pre-clinical studies in this area lead us to hypothesize that these differences may be secondary to gut permeability. Future studies are needed to confirm this hypothesis.

Novel findings of this study include the differences in $\beta$-diversity as well as in the number of species with DNA expressed. In particular, DNA from the Bacteroidetes phylum differed by age and was significantly correlated with indices of inflammation. Given the lower abundance of Bacteroidetes DNA among older adults - these data could suggest a causal relationship between microbial DNA community composition and lower IGF1/higher inflammatory cytokines observed with advanced age. Though speculative, as a dominant microbial community, it is possible that reductions in circulating concentrations indicate increases in other potentially more reactive communities.

Another novel finding of the study is association of serum microbial DNA abundances with IGF1. 
Table 2 Serum microbiome composition at the class level (25 most common OTUs)

\begin{tabular}{|c|c|c|c|}
\hline & Young adults & Older adults & $p$ value for group \\
\hline Firmicutes_Clostridia & $34.8 \pm 14.3$ & $34.5 \pm 15.4$ & 0.932 \\
\hline Bacteroidetes_Bacteroidia & $18.2 \pm 4.7$ & $13.6 \pm 5.0$ & $0.003 *$ \\
\hline Firmicutes_Bacilli & $12.5 \pm 7.0$ & $11.4 \pm 4.7$ & 0.831 \\
\hline Proteobacteria_Gammaproteobacteria & $9.6 \pm 6.3$ & $7.7 \pm 3.2$ & 0.580 \\
\hline Actinobacteria_Actinobacteria & $4.9 \pm 6.4$ & $7.6 \pm 7.4$ & 0.093 \\
\hline Firmicutes_Erysipelotrichi & $2.3 \pm 1.6$ & $8.6 \pm 13.4$ & 0.023 \\
\hline Fusobacteria_Fusobacteriia & $5.9 \pm 4.6$ & $3.4 \pm 3.6$ & 0.035 \\
\hline Proteobacteria_Betaproteobacteria & $2.7 \pm 0.9$ & $3.4 \pm 2.3$ & 0.496 \\
\hline Proteobacteria_Alphaproteobacteria & $2.3 \pm 2.3$ & $2.4 \pm 1.8$ & 0.702 \\
\hline Verrucomicrobia_Verrucomicrobiae & $1.9 \pm 0.8$ & $2.3 \pm 1.3$ & 0.217 \\
\hline Proteobacteria_Epsilonproteobacteria & $0.8 \pm 0.4$ & $0.6 \pm 0.4$ & 0.085 \\
\hline Actinobacteria_Coriobacteriia & $0.5 \pm 0.2$ & $0.6 \pm 0.3$ & 0.120 \\
\hline Bacteroidetes_Flavobacteriia & $0.5 \pm 0.2$ & $0.6 \pm 0.7$ & 0.898 \\
\hline Cyanobacteria_Chloroplast & $0.5 \pm 0.4$ & $0.7 \pm 0.5$ & 0.173 \\
\hline Tenericutes_Mollicutes & $0.7 \pm 0.5$ & $0.5 \pm 0.4$ & $0.003 *$ \\
\hline Proteobacteria_Deltaproteobacteria & $0.4 \pm 0.3$ & $0.4 \pm 0.3$ & 0.865 \\
\hline Bacteria_Other_Other & $0.3 \pm 0.2$ & $0.2 \pm 0.2$ & $0.004 *$ \\
\hline Bacteria_SR1_unknown & $0.2 \pm 0.2$ & $0.1 \pm 0.2$ & 0.016 \\
\hline Bacteroidetes_Cytophagia & $0.04 \pm 0.04$ & $0.25 \pm 0.49$ & $0.003 *$ \\
\hline Cyanobacteria_Synechococcophycideae & $0.07 \pm 0.04$ & $0.16 \pm 0.22$ & 0.328 \\
\hline Deferribacteres_Deferribacteres & $0.10 \pm 0.10$ & $0.10 \pm 0.08$ & 0.686 \\
\hline Firmicutes_Other & $0.11 \pm 0.07$ & $0.06 \pm 0.05$ & $0.011 *$ \\
\hline Spirochaetes_Leptospirae & $0.10 \pm 0.08$ & $0.04 \pm 0.06$ & $0.001 *$ \\
\hline Actinobacteria_Acidimicrobiia & $0.03 \pm 0.03$ & $0.04 \pm 0.06$ & 0.045 \\
\hline Cyanobacteria_Oscillatoriophycideae & $0.02 \pm 0.03$ & $0.10 \pm 0.20$ & 0.034 \\
\hline
\end{tabular}

All values (mean $\pm \mathrm{SD}$ ) indicate relative abundance $(\%)$

*Statistically significant after correction for false discovery rate

Though typically known for its potent anabolic properties, IGF1 also has tremendous relevance to the human immune system. It is well documented that inflammatory cytokines attenuate IGF1 production (Maggio et al. 2013; Rajpathak et al. 2008), but IGF1 also plays an important role in regulating innate and acquired immunity - including the production of inflammatory cytokines (Heemskerk et al. 1999). Clinical data have recently implicated low IGF1 in flare-ups of inflammatory bowel disease (Krakowska-Stasiak et al. 2017), while basic studies have demonstrated that IGF1 directly inhibits pro-inflammatory cytokines in multiple animal cell types (Ji et al. 2017; Onnureddy et al. 2015), inducing LPS-induced cytokine expression (Onnureddy et al. 2015). This latter finding may have important implications for present findings, as microbial LPS may stimulate inflammatory cytokine production. Moreover, recent data reported that gut microbiota can stimulate IGF1 (Yan et al. 2016). Despite these links, the present data should not be over-interpreted as they do not provide any indication of directional causality. However, they do suggest that further follow-up may be warranted given the strength of associations and the aforementioned recent literature in this area.

Notably, dietary intake - including intake of dietary fiber-and fitness differed between young and older adults. Though these are differences commonly observed between young and older adults, these findings are important in the present context as diet and physical activity/exercise are among the primary factors known 

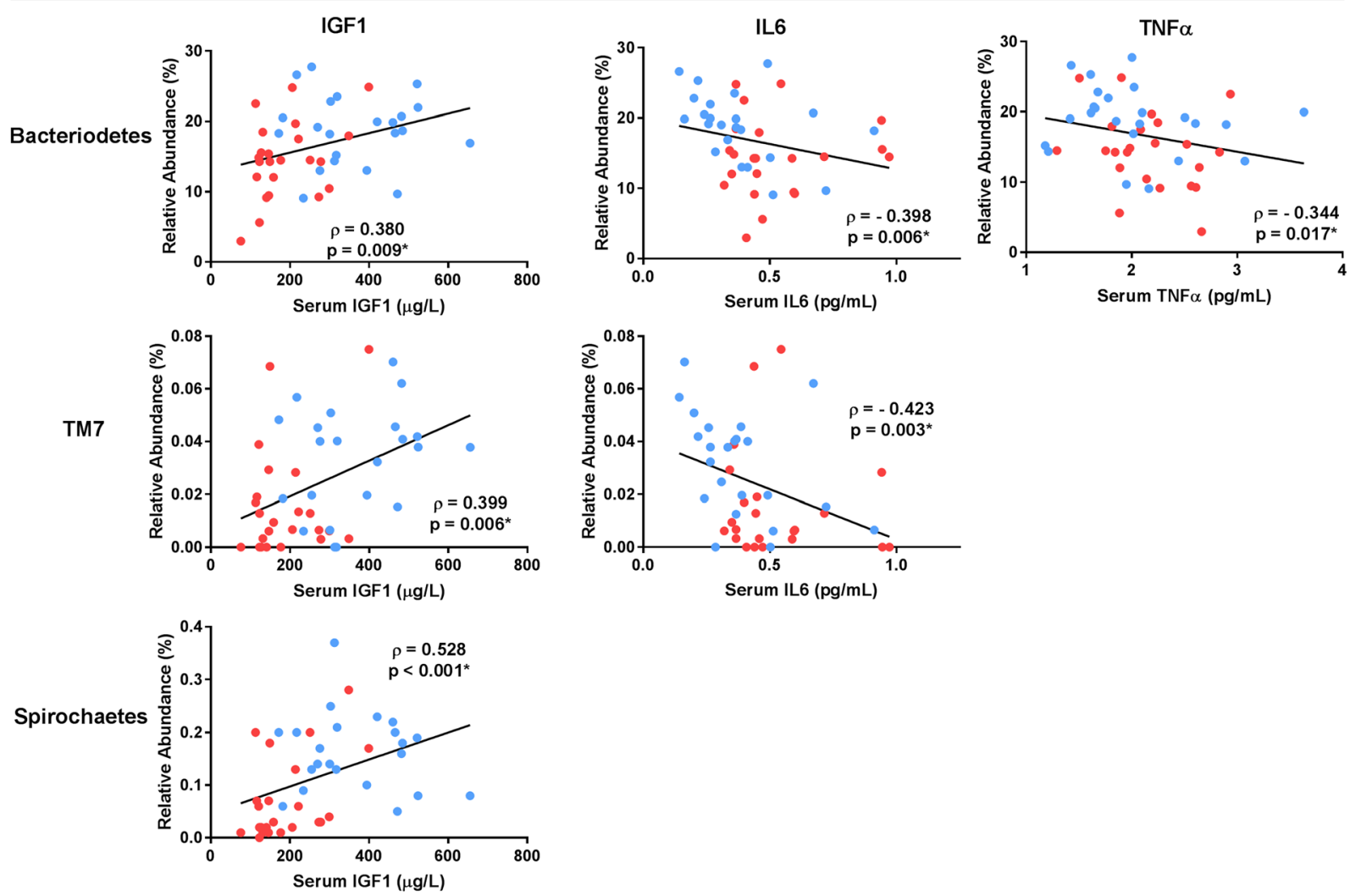

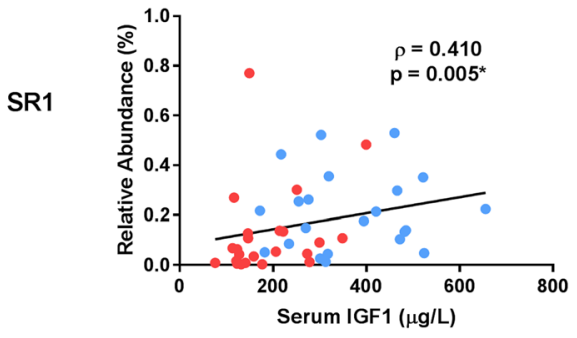

Fig. 4 Microbial DNA populations at the phylum level significantly differing in abundance between young and older adults and correlated with indices of inflammation. Correlation coefficients reflect the Spearman rho comparison. Asterisk indicates statistical

to influence gut microbiota communities (Campbell and Wisniewski 2017; Chen et al. 2014; O’Sullivan et al. 2015; Pallister and Spector 2016). To our knowledge, no data exist to directly indicate that diet or exercise can alter systemic bacterial DNA expression. However, both high-fat meals and highly vigorous exercise are known to be capable of inducing intestinal permeability, bacterial translocation, and even transient endotoxemia (Costa et al. 2017; Kelly et al. 2012). It is unclear at present how these factors might contribute to age-related differences in serum microbiome profiles, but these factors are important to consider for proper interpretation of study findings and in moving forward to causal studies. significance after correcting for multiple comparisons via false discovery rate. Data points are colored separately to indicate young (blue) and older (red) adults

As with any study, the present investigation is not without limitations. For instance, as noted above, the $16 \mathrm{~S}$ microbiome analysis does not discriminate between microbial DNA fragment and intact microbes.

However, as noted, previous studies have shown that even bacterial DNA fragments are capable of stimulating immune reactions based on their foreign structure (Hacker et al. 2002; Muruve et al. 2008; Nikkari et al. 2001). Again, it is possible that differences in serum microbial DNA expression may be influenced by exercise (as evidenced by fitness) or diet which are important regulators of the intestinal microbiome. However, this is 
IGF1

Bacterioidia
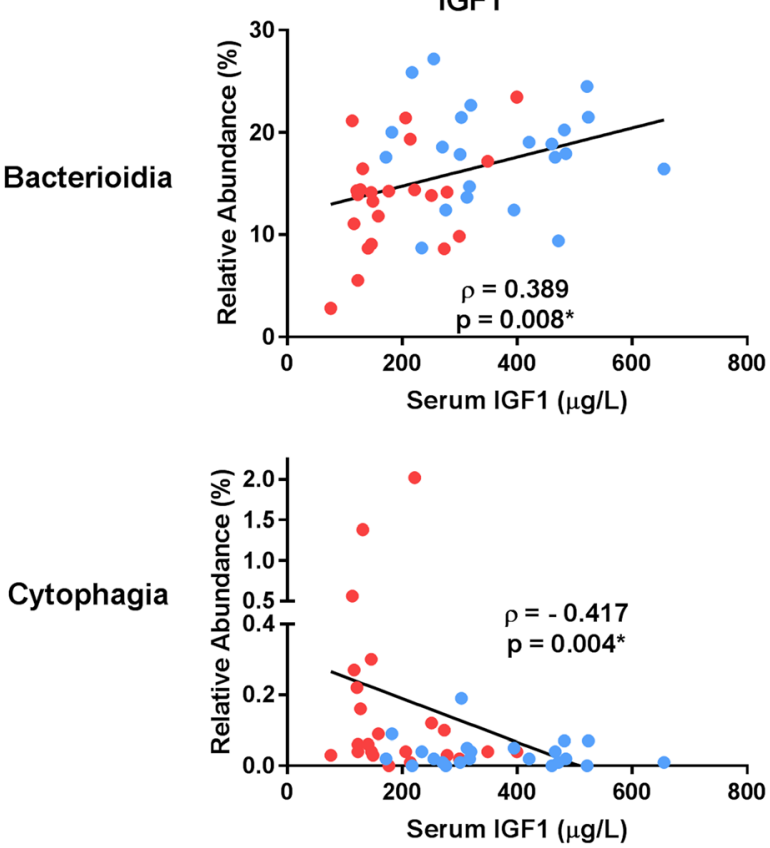

IL6

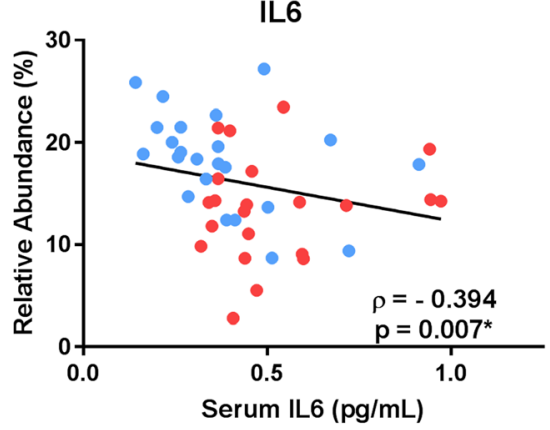

Fig. 5 Microbial DNA populations at the class level significantly differing in abundance between young and older adults and correlated with indices of inflammation. Correlation coefficients reflect the Spearman rho comparison. Asterisk indicates statistical significance after correcting for multiple comparisons via false discovery rate. Data points are colored separately to indicate young (blue) and older (red) adults

disease and not obese (body mass index $<30 \mathrm{~kg} / \mathrm{m}^{2}$ ). All subjects completed health history questionnaires, and older adults passed a comprehensive physical exam and a diagnostic exercise stress test with 12-lead ECG to confirm health status. All participants were also assessed for body composition via dual $\mathrm{x}$-ray absorptiometry and for aerobic fitness (i.e., $\mathrm{VO}_{2} \mathrm{max}$ ) via a maximal exercise challenge with expired gases as further indicators of overall health status. Habitual dietary intake was assessed via 4-day food records analyzed using Nutrition Data Systems for Research (NDSR) software (Nutrition Coordinating Center, University of Minnesota, Minneapolis, MN). Prior to participation, all participants provided written informed based on documents approved by Institutional Review Boards of the University of Alabama at Birmingham (UAB) and Birmingham Veterans Affairs Medical Center.

Blood collection and inflammatory analyses Venous blood was collected and spun down to obtain serum using standard clinical practices. Serum IL6 and TNF $\alpha$ were determined using a Meso Scale Discovery (MSD; Rockville, MD) Quick Plex SQ 120 imager using electrochemiluminescence technology. Minimum 
sensitivity for the IL6 assay was $0.07 \mathrm{pg} / \mathrm{mL}$, while sensitivity was $0.09 \mathrm{pg} / \mathrm{mL}$ for TNF $\alpha$. Intra-assay coefficients of variation (CV) were 7.84 and $7.67 \%$, and inter-assay coefficients were 5.78 and $2.5 \%$ for IL6 and TNF $\alpha$, respectively. IGF1 was assessed via immunoradiometric assay (Diagnostic Systems Laboratories, Webster, TX). The inter-assay $\mathrm{CV}$, intra-assay $\mathrm{CV}$, and assay sensitivity for IGF1 were $9.43,3.48$, and $4.89 \mathrm{ng} / \mathrm{mL}$, respectively.

Microbiome analyses-16S PCR amplification The $16 \mathrm{~S}$ V4 analysis was done as previously described (Kumar et al. 2014). DNA was extracted from serum samples with the ZR Fecal DNA Miniprep Kit (Zymo Research, Irvine, CA) (Kumar et al. 2014). PCR was used with unique bar-coded primers to amplify the V4 region of the 16S rRNA gene to create an "amplicon library" from individual samples as described by Kumar et al. (2014). Cycling conditions for the PCR reactions were as follows: initial denature $94{ }^{\circ} \mathrm{C}$ for $1 \mathrm{~min}$ followed by 32 cycles of $94{ }^{\circ} \mathrm{C}$ for $30 \mathrm{~s}, 50{ }^{\circ} \mathrm{C}$ for $1 \mathrm{~min}$, $65{ }^{\circ} \mathrm{C}$ for $1 \mathrm{~min}$, and a final extension of $65{ }^{\circ} \mathrm{C}$ for $3 \mathrm{~min}$. The entire PCR reaction was electrophoresed on a $1.0 \%$ agarose/Tris-borate-EDTA gel. The PCR product (approximately 250 base pairs) was visualized by UV illumination. The band was excised and purified from the agarose using Qiagen QIAquick Gel Extraction Kit according to the manufacturer's instructions.

The PCR products were then sequenced using the Illumina MiSeq platform (Kumar et al. 2014). Paired end reads of approximately $250 \mathrm{bp}$ from the V4 region of $16 \mathrm{~S}$ rDNA were analyzed. The samples were first quantitated using Pico Green, adjusted to a concentration of $4 \mathrm{nM}$ then used for sequencing on the Illumina MiSeq (Kumar et al. 2014). Fastq conversion of the raw data files was performed following de-multiplexing. Quality control of the fastq files was performed which was then subject to quality assessment and filtering using the FASTX toolkit (FASTX). The remainder of the steps was performed using the Quantitative Insight into Microbial Ecology (QIIME) suite, version 1.8 (Kumar et al. 2014; Lozupone et al. 2007; Navas-Molina et al. 2013). One sample was removed from analysis due to failing quality control procedures.
Microbiome analyses-sequence data analysis and composition The sequence data covered the $16 \mathrm{~S}$ rRNA V4 region with a PCR product length of 255 bases and 250 base paired-end reads. Since the overlap between fragments was approximately 245 bases, the information from both ends of the paired reads was merged to generate a single high-quality read using the module "fastq_mergepairs" of USEARCH (Edgar 2010). Read pairs with an overlap of less than 50 bases or with too many mismatches (> $20)$ in the overlapping region were discarded. Chimeric sequences were also filtered using the "identify_chimeric_seqs.py" module of USEARCH (Edgar 2010). Overall, read quality was assessed before and after filtering using FASTQC (FASTQC. http://Www. bioinformatics.babraham.ac. uk/projects/fastqc/). The QIIME data analysis package was used for subsequent $16 \mathrm{~S}$ rRNA data analysis (Caporaso et al. 2010a, b). Sequences were grouped into operational taxonomic units (OTUs) using the clustering program UCLUST at a similarity threshold of $0.97 \%$ (Edgar 2010). The Ribosomal Database Program (RDP) classifier was used to make taxonomic assignments (to the genus and/or species level) for all OTUs at confidence threshold of $80 \%$ (0.8) (Wang et al. 2007). The RDP classifier was trained using the Greengenes (v13_8) 16S rRNA database (McDonald et al. 2012).

The resulting OTU table included all OTUs, their taxonomic identification, and abundance information. OTUs whose average abundance was less than $0.0005 \%$ were filtered out. OTUs were then grouped together to summarize taxon abundance at different hierarchical levels of classification (e.g., phylum, class, etc). Multiple sequence alignment of OTUs was performed with PyNAST (Caporaso et al. 2010a, b). Alpha diversity (diversity within the samples) was calculated using Shannon's diversity matrix which measures both richness (number of OTUs/ species present in a sample) and evenness (relative abundance of different OTUs/species and their even distribution in a sample) (Jost 2007), as implemented in QIIME (Caporaso et al. 2010a, b). Beta diversity (diversity between the samples) was measured using unweighted Unifrac analysis (Lozupone and Knight 2005). Principal coordinate analysis (PCoA) was performed by QIIME to visualize the dissimilarity matrix between all samples, such that samples that were more similar were closer in space 
than samples that were more divergent. A 3D PCoA plot was generated using EMPEROR (Vazquez-Baeza et al. 2013).

Statistical analysis All data were evaluated for normality and homogeneity of variance prior to determination of descriptive statistics and comparative analyses. Group comparisons for demographic, dietary, and inflammatory data were performed using Student's $t$ tests for independent samples. The observed species metric of $\alpha$-diversity was assessed using Student's $t$ test. Other indices of $\alpha$-diversity were assessed via the MannWhitney test. A $p$ value of $<0.05$ was utilized to identify differences in descriptive data between groups. Comparison of microbial abundances between groups, both at the phylum and class levels, were analyzed using the non-parametric Mann-Whitney test. For the class level, only the 25 most common OTUs were evaluated due to the low abundance of other OTUs. A significance level of $p<0.05$ was utilized for initial identification of OTUs of interest, with final determination of significance established after correcting for false discovery rate (FDR) according to the method of Benjamini and Hochberg (1995). Following comparative analyses, correlational analyses were performed among the inflammatory parameters and those OTUs identified (adjusted for FDR) as significantly differing in relative abundance between age groups. Correlation coefficients were calculated using the Spearman procedure. Correlations with $p$ values $<0.05$ were flagged, with final determination of significance established after correcting for FDR.

Acknowledgements We are thankful to all study participants for their contributions. This work was supported by grants from the National Institutes of Health, including the National Institute on Aging (R01AG017896 to MMB, R01AG054538 to TWB), the National Center for Medical Rehabilitation Research (P2CHD086851 to MMB), and the Department of Veterans Affairs (Merit Grant to MMB). The following are acknowledged for their support of the Microbiome Resource at the University of Alabama at Birmingham: School of Medicine, Comprehensive Cancer Center (P30 CA013148), Center for Clinical Translational Science (UL1TR001417), Microbiome Center and Heflin Center for Genomic Sciences. The work was also supported by the UAB Metabolism Core Laboratory, funded in part by NIH grants P30DK56336, P30DK079626, and U4TR001368.

Compliance with ethical standards Prior to participation, all participants provided written informed based on documents approved by Institutional Review Boards of the University of Alabama at Birmingham (UAB) and Birmingham Veterans Affairs Medical Center.
Open Access This article is distributed under the terms of the Creative Commons Attribution 4.0 International License (http:// creativecommons.org/licenses/by/4.0/), which permits unrestricted use, distribution, and reproduction in any medium, provided you give appropriate credit to the original author(s) and the source, provide a link to the Creative Commons license, and indicate if changes were made.

\section{References}

Belkaid Y, Naik S (2013) Compartmentalized and systemic control of tissue immunity by commensals. Nat Immunol 14(7):646653. https://doi.org/10.1038/ni.2604

Benjamini, Y., \& Hochberg, Y. (1995). Controlling the false discovery rate: a practical and powerful approach to multiple testing. J. Royal Stat. Soc., 289-290-300

Biagi E, Nylund L, Candela M, Ostan R, Bucci L, Pini E et al (2010) Through ageing, and beyond: gut microbiota and inflammatory status in seniors and centenarians. PLoS One 5(5):e10667. https://doi.org/10.1371/journal.pone.0010667 [doi]

Brenchley JM, Price DA, Schacker TW, Asher TE, Silvestri G, Rao S et al (2006) Microbial translocation is a cause of systemic immune activation in chronic HIV infection. Nat Med 12(12):1365-1371

Buford, T. W. (2017). (Dis)trust your gut: the gut microbiome in age-related inflammation, health, and disease Microbiome, 5(1), 80-017-0296-0. https://doi.org/10.1186/s40168-0170296-0

Campbell SC, Wisniewski PJ 2nd. (2017) Exercise is a novel promoter of intestinal health and microbial diversity. Exerc Sport Sci Rev 45(1):41-47. https://doi.org/10.1249 /JES.0000000000000096

Caporaso JG, Bittinger K, Bushman FD, DeSantis TZ, Andersen GL, Knight R (2010a) PyNAST: a flexible tool for aligning sequences to a template alignment. Bioinformatics (Oxford, England) 26(2):266-267. https://doi.org/10.1093 /bioinformatics/btp636

Caporaso JG, Kuczynski J, Stombaugh J, Bittinger K, Bushman FD, Costello EK, Fierer N, Peña AG, Goodrich JK, Gordon JI, Huttley GA, Kelley ST, Knights D, Koenig JE, Ley RE, Lozupone CA, McDonald D, Muegge BD, Pirrung M, Reeder J, Sevinsky JR, Turnbaugh PJ, Walters WA, Widmann J, Yatsunenko T, Zaneveld J, Knight R (2010b) QIIME allows analysis of high-throughput community sequencing data. Nat Methods 7(5):335-336. https://doi. org/10.1038/nmeth.f.303 [doi]

Chen J, He X, Huang J (2014) Diet effects in gut microbiome and obesity. J Food Sci 79(4):R442-R451. https://doi. org/10.1111/1750-3841.12397

Claesson MJ, Jeffery IB, Conde S, Power SE, O'Connor EM, Cusack S et al (2012) Gut microbiota composition correlates with diet and health in the elderly. Nature 488(7410):178184. https://doi.org/10.1038/nature11319 [doi]

Clark RI, Salazar A, Yamada R, Fitz-Gibbon S, Morselli M, Alcaraz J, Rana A, Rera M, Pellegrini M, Ja WW, Walker DW (2015) Distinct shifts in microbiota composition during 
drosophila aging impair intestinal function and drive mortality. Cell Rep 12(10):1656-1667. https://doi.org/10.1016/j. celrep.2015.08.004

Costa RJS, Snipe RMJ, Kitic CM, Gibson PR (2017) Systematic review: exercise-induced gastrointestinal syndromeimplications for health and intestinal disease. Aliment Pharmacol Ther 46(3):246-265. https://doi.org/10.1111 /apt. 14157

Dinakaran V, Rathinavel A, Pushpanathan M, Sivakumar R, Gunasekaran P, Rajendhran J (2014) Elevated levels of circulating DNA in cardiovascular disease patients: metagenomic profiling of microbiome in the circulation. PloS One 9(8):e105221. https://doi.org/10.1371/journal. pone. 0105221

Edgar RC (2010) Search and clustering orders of magnitude faster than BLAST. Bioinformatics (Oxford, England) 26(19): 2460-2461. https://doi.org/10.1093/bioinformatics/btq461

FASTQC. http://Www.bioinformatics.babraham.ac. uk/projects/fastqc/

Frances R, Munoz C, Zapater P, Uceda F, Gascon I, Pascual S et al (2004) Bacterial DNA activates cell mediated immune response and nitric oxide overproduction in peritoneal macrophages from patients with cirrhosis and ascites. Gut 53(6): 860-864

Hacker G, Redecke V, Hacker H (2002) Activation of the immune system by bacterial CpG-DNA. Immunology 105(3):245-251

Heemskerk VH, Daemen MA, Buurman WA (1999) Insulin-like growth factor-1 (IGF-1) and growth hormone (GH) in immunity and inflammation. Cytokine Growth Factor Rev 10(1):5-14

Human Microbiome Project Consortium (2012) A framework for human microbiome research. Nature 486(7402):215-221. https://doi.org/10.1038/nature11209

Jeffery IB, Lynch DB, O'Toole PW (2016) Composition and temporal stability of the gut microbiota in older persons. The ISME Journal 10(1):170-182. https://doi.org/10.1038 /ismej.2015.88

Ji Y, Duan W, Liu Y, Liu Y, Liu C, Li Y et al (2017) IGF1 affects macrophage invasion and activation and TNF-alpha production in the sciatic nerves of female SOD1G93A mice. Neurosci Lett 668:1-6

Jost L (2007) Partitioning diversity into independent alpha and beta components. Ecology 88(10):2427-2439

Kelly CJ, Colgan SP, Frank DN (2012) Of microbes and meals: the health consequences of dietary endotoxemia. Nutr Clin Pract: Off Publ Am Soc Parenter Enter Nutr 27(2):215-225. https://doi.org/10.1177/0884533611434934

Krakowska-Stasiak M, Cibor D, Domagala-Rodacka R, Salapa K, Szczeklik K, Owczarek D (2017) Insulinlike growth factor system in remission and flare of inflammatory bowel diseases. Pol Arch Intern Med 127(12):832-839. https://doi. org/10.20452/pamw.4136

Kumar R, Eipers P, Little RB, Crowley M, Crossman DK, Lefkowitz EJ, Morrow CD (2014) Getting started with microbiome analysis: sample acquisition to bioinformatics. Curr Protoc Hum Genet 82:18.8.1-18.829. https://doi. org/10.1002/0471142905.hg1808s82

Kwan, B. C., Chow, K. M., Leung, C. B., Law, M. C., Cheng, P. M., Yu, V., ... Szeto, C. C. (2013) Circulating bacterialderived DNA fragments as a marker of systemic inflammation in peritoneal dialysis. Nephrol Dial Transplant: Off Publ Eur Dial Transplant Assoc Eur Ren Assoc, 28(8), 2139-2145. https://doi.org/10.1093/ndt/gft100

Lozupone C, Knight R (2005) UniFrac: a new phylogenetic method for comparing microbial communities. Appl Env Microbiol 71(12):8228-8235

Lozupone CA, Hamady M, Kelley ST, Knight R (2007) Quantitative and qualitative beta diversity measures lead to different insights into factors that structure microbial communities. Appl Environ Microbiol 73(5):1576-1585

Maggio M, De Vita F, Lauretani F, Butto V, Bondi G, Cattabiani C et al (2013) IGF-1, the cross road of the nutritional, inflammatory and hormonal pathways to frailty. Nutrients $5(10)$ : 4184-4205. https://doi.org/10.3390/nu5104184

Man AL, Bertelli E, Rentini S, Regoli M, Briars G, Marini M et al (2015) Age-associated modifications of intestinal permeability and innate immunity in human small intestine. Clin Sci (London, England: 1979) 129(7):515-527. https://doi. org/10.1042/CS20150046

McDonald D, Price MN, Goodrich J, Nawrocki EP, DeSantis TZ, Probst A, Andersen GL, Knight R, Hugenholtz P (2012) An improved greengenes taxonomy with explicit ranks for ecological and evolutionary analyses of bacteria and archaea. ISME J 6(3):610-618. https://doi.org/10.1038 /ismej.2011.139 [doi]

Muruve DA, Petrilli V, Zaiss AK, White LR, Clark SA, Ross PJ et al (2008) The inflammasome recognizes cytosolic microbial and host DNA and triggers an innate immune response. Nature 452(7183):103-107. https://doi.org/10.1038 /nature06664 [doi]

Navas-Molina JA, Peralta-Sanchez JM, Gonzalez A, McMurdie PJ, Vazquez-Baeza Y, Xu Z et al (2013) Advancing our understanding of the human microbiome using QIIME. Methods Enzymol 531:371-444. https://doi.org/10.1016 /B978-0-12-407863-5.00019-8 [doi]

Nicoletti C (2015) Age-associated changes of the intestinal epithelial barrier: local and systemic implications. Expert Rev Gastroenterol Hepatol 9(12):1467-1469. https://doi. org/10.1586/17474124.2015.1092872

Nikkari S, McLaughlin IJ, Bi W, Dodge DE, Relman DA (2001) Does blood of healthy subjects contain bacterial ribosomal DNA? J Clin Microbiol 39(5):1956-1959. https://doi. org/10.1128/JCM.39.5.1956-1959.2001

Onnureddy, K., Ravinder Onteru, S. K., \& Singh, D. (2015). IGF1 attenuates LPS induced pro-inflammatory cytokines expression in buffalo (Bubalus bubalis) granulosa cells. Mol Immunol, 64(1), 136-143. https://doi.org/10.1016/j. molimm.2014.11.008 [doi]

O'Sullivan O, Cronin O, Clarke SF, Murphy EF, Molloy MG, Shanahan F, Cotter PD (2015) Exercise and the microbiota. Gut Microbes 6(2):131-136. https://doi.org/10.1080 $/ 19490976.2015 .1011875$

Pallister T, Spector TD (2016) Food: a new form of personalised (gut microbiome) medicine for chronic diseases? J R Soc Med 109(9):331-336. https://doi.org/10.1177 /0141076816658786

Qi, Y., Goel, R., Kim, S., Richards, E. M., Carter, C. S., Pepine, C. J., ... Buford, T. W. (2017). Intestinal permeability biomarker zonulin is elevated in healthy aging. J Am Med Dir Assoc, 18(9), 810.e1-810.e4 
Rajpathak SN, McGinn AP, Strickler HD, Rohan TE, Pollak M, Cappola AR, Kuller L, Xue XN, Newman AB, Strotmeyer ES, Psaty BM, Kaplan RC (2008) Insulin-like growth factor(IGF)-axis, inflammation, and glucose intolerance among older adults. Growth Hormon IGF Res: Off J Growth Hormon Res Soc Int IGF Res Soc 18(2):166-173

Rampelli S, Candela M, Turroni S, Biagi E, Collino S, Franceschi $\mathrm{C}$ et al (2013) Functional metagenomic profiling of intestinal microbiome in extreme ageing. Aging 5(12):902-912

Rera M, Clark RI, Walker DW (2012) Intestinal barrier dysfunction links metabolic and inflammatory markers of aging to death in drosophila. Proc Natl Acad Sci U S Am 109(52): 21528-21533. https://doi.org/10.1073/pnas.1215849110

Santiago A, Pozuelo M, Poca M, Gely C, Nieto JC, Torras X, Román E, Campos D, Sarrabayrouse G, Vidal S, AlvaradoTapias E, Guarner F, Soriano G, Manichanh C, Guarner C (2016) Alteration of the serum microbiome composition in cirrhotic patients with ascites. Sci Rep 6:25001. https://doi. org/10.1038/srep25001 [doi]

Spadoni I, Zagato E, Bertocchi A, Paolinelli R, Hot E, Di Sabatino A et al (2015) A gut-vascular barrier controls the systemic dissemination of bacteria. Science (New York, NY) 350(6262):830-834. https://doi.org/10.1126/science. aad0135 [doi]

Thevaranjan N, Puchta A, Schulz C, Naidoo A, Szamosi JC, Verschoor CP et al (2017) Age-associated microbial dysbiosis promotes intestinal permeability, systemic inflammation, and macrophage dysfunction. Cell Host Microbe 21(4):455-466.e4

Vazquez-Baeza Y, Pirrung M, Gonzalez A, Knight R (2013) EMPeror: a tool for visualizing high-throughput microbial community data. GigaScience 2(1):16-217X-2-16. https://doi.org/10.1186/2047-217X-2-16

Wang Q, Garrity GM, Tiedje JM, Cole JR (2007) Naive bayesian classifier for rapid assignment of rRNA sequences into the new bacterial taxonomy. Appl Environ Microbiol 73(16): 5261-5267

Yan J, Herzog JW, Tsang K, Brennan CA, Bower MA, Garrett WS et al (2016) Gut microbiota induce IGF-1 and promote bone formation and growth. Proc Natl Acad Sci U S A 113(47): E7554-E7563 